\title{
Panic Reactions to Terrorist Attacks and Probable Posttraumatic Stress Disorder in Adolescents*
}

\author{
Betty Pfefferbaum \\ University of Oklahoma Health Sciences Center \\ Jennifer Stuber \\ New York Academy of Medicine \\ Sandro Galea \\ University of Michigan School of Public Health \\ Gerry Fairbrother \\ University of Cincinnati
}

\begin{abstract}
A number of factors, including subjective reactions and appraisal of danger, influence one's reaction to a traumatic event. This study used telephone survey methodology to examine adolescent and parent reactions to the 2001 World Trade Center attacks 6 to 9 months after they occurred. The prevalence of probable posttraumatic stress disorder (PTSD) in adolescents was $12.6 \%$; $26.2 \%$ met study criteria for probable subthreshold PTSD. A probable peri-event panic attack in adolescents was strongly associated with subsequent probable PTSD and probable subthreshold PTSD. This study suggests that the early identification of peri-event panic attacks following mass traumatic events may provide an important gateway to intervention in the subsequent development of PTSD. Future studies should use longitudinal designs to examine the course and pathogenic pathways for the development of panic, PTSD, and other anxiety disorders after exposure to disasters.
\end{abstract}

The September 11, 2001 attacks on the World Trade Center were horrifying. Thousands were killed or injured and many more fled from the smoke, ash, and devastation. Children and adolescents, like adults, were exposed through physical proximity, interpersonal relationships to victims, and media coverage of the disaster. Interested in studying the reactions of adolescents, we examined exposure and other factors thought to influence outcome, including peri-event subjective reaction and appraisal of danger (Asarnow et al., 1999; Pfefferbaum et al., 2002;
Schwarz \& Kowalski, 1991) and characteristics of the family (Green et al., 1991; McFarlane, 1987) and social environment (La Greca, Silverman, Vernberg, \& Prinstein, 1996).

\section{SUBJECTIVE RERCTIONS AND PANIC}

Subjective reactions to disasters range from experiences of anxiety, fear, and worry (Lonigan, Shannon, Taylor, Finch, $\&$ Sallee, 1994; Pfefferbaum et al., 2002) to perceptions of

\footnotetext{
*This article was edited by the journal's previous editor, Dean G. Kilpatrick.

Correspondence concerning this article should be addressed to: Betty Pfefferbaum, Department of Psychiatry and Behavioral Sciences, University of Oklahoma Health Sciences Center, 920 Stanton L. Young Boulevard, WP-3470, Oklahoma City, OK 73104. E-mail: betty-pfefferbaum@ouhsc.edu.

(C) 2006 International Society for Traumatic Stress Studies. Published online in Wiley InterScience (www.interscience.wiley.com) DOI: 10.1002/jts.20118
} 
life threat (Green et al., 1991) and panic (Udwin, Boyle, Yule, Bolton, \& O'Ryan, 2000), the focus of this study. Panic represents the basic fear reaction triggered in situations of danger and is associated with the fight-or-flight response (Jones \& Barlow 1990). It includes cognitive and somatic symptoms distinguished from other anxiety conditions, in part, by subjective intensity and abruptness (Barlow, 2002). Panic disorder and posttraumatic stress disorder (PTSD) share a number of signs, symptoms, and cognitions and may appear as comorbid conditions. Individuals with panic disorder often report a history of stressful life events and people exposed to trauma may develop both panic disorder and PTSD (Falsetti et al., 1995). Endocrine and sleep studies evidence similarities in the two disorders (Falsetti et al., 1995; Pitman, 1993).

The etiology of panic, which can occur in the absence of actual danger, is the subject of some debate with theories focusing on biological and psychological factors including a genetic predisposition and cognitive and social determinants (Falsetti et al., 1995). Falsetti and colleagues (1995) suggest that panic disorder may occur in the absence of biological or psychological vulnerability in individuals who have experienced traumatic stress noting that external (e.g., places, situations, and objects) and internal (e.g., emotions, arousal, and cognitions) cues associated with the event might trigger later attacks. Some of these attacks may appear to be "out of the blue" if the individual does not appreciate the connection to the event. Chronic hyperarousal and hypervigilance associated with PTSD may increase vulnerability to future panic attacks by lowering the threshold of arousal needed to trigger or maintain future panic attacks. Avoidance-efforts to decrease the chance of experiencing distressing psychological and physiological reactions-may strengthen the association between cues and responses through escape-avoidance learning and lack of extinction, thus maintaining a cycle of hyperarousal and panic attacks.

While panic has been linked to trauma exposure, the association between panic and trauma exposure has received relatively little attention in the disaster literature. In adults, experiencing a panic attack during or shortly after the September 11, 2001, attacks predicted probable PTSD in residents of lower Manhattan surveyed 5 to 8 weeks later (Galea et al., 2002). Fear of dying and feelings of panic were among the subjective reactions associated with the development of PTSD in young adult survivors of a shipping disaster during their adolescence (Udwin et al., 2000).

Panic attacks are not uncommon in adolescents (Ollendick, 1998; Ollendick, Mattis, \& King, 1994). Hayward, Killen, Kraemer, and Taylor (2000) found a lifetime prevalence of $10.2 \%$ for panic attacks in a nonclinical adolescent sample. The study of panic in adolescents is important, in part, because rates of onset peak during adolescence (Hayward et al., 1997), because early onset may increase risk for morbidity (Black \& Robbins, 1990), and because panic attacks may be associated with other conditions and with considerable distress (Ollendick, 1998). Thus, the relationship between trauma exposure and panic warrants attention in this age group.

\section{FAMILY AND SOCIAL INFLUENCE}

Studies have documented a positive relationship in posttraumatic stress symptoms in parent-child dyads (Breton, Valla, \& Lambert, 1993; Earls, Smith, Reich, \& Jung, 1988), but relatively few studies have examined both parent and child reactions contemporaneously. Parental functioning and family atmosphere after the Buffalo Creek disaster predicted PTSD symptoms in young children and adolescents (Green et al., 1991). Availability of social support attenuated the impact of a hurricane in children (La Greca et al., 1996). Another aspect of the postdisaster environment, exposure to media coverage of a disaster, has also been linked to posttraumatic stress reactions in children (Fairbrother et al., 2003; Pfefferbaum et al., 1999; Schuster et al., 2001).

\section{FRAMEWORH FOR THIS STUDY}

To explore factors associated with adolescent posttraumatic stress reactions, we surveyed a community sample of adolescents and their parents living in New York City at the time of the September 11 attacks. Our analysis focused on the experience of a panic attack in the immediate aftermath 
of the disaster as a potential predictor of posttraumatic stress reactions and also examined aspects of the recovery environment including the reactions of parents, adolescent perceptions of pre-disaster social support, and exposure to television coverage of the incident.

\section{METHOD}

Interviews with parents and adolescents were conducted by interviewers using a computer-assisted telephone interview system in English, Spanish, or Chinese. All interviewers were professional interviewers, trained in the administration of this specific instrument by the study investigators. Adult respondents were identified for interview through a random digit-dial telephone survey of 2,752 adults (18 years of age or older) conducted in the New York City Metropolitan Area between March 25 and June 25, 2002, 6 to 9 months after the September 11 attacks. A random adult in the household was selected for interview using the last birthday procedure (the adult with the most recent birthday). The overall response rate among eligible persons was 56\% (American Association for Public Opinion Research, 2000). The sample was comparable to the New York City population in terms of age, gender, and race (Galea et al., 2003).

All adult respondents were asked if they were the parent or primary caretaker of a child between the ages of 4 and 17 years who was living in the household; 634 adults responded affirmatively and were asked additional questions about a child in the household. Of those, 529 agreed (10 surveys were incomplete) for a response rate of $85 \%$. Parents who had more than one child in this age range were asked about the child who had most recently celebrated a birthday. Among the 529 participating adults were 249 parents of adolescents between 12 and 17 years of age. Parental assent was obtained to interview the adolescent. Forty-eight parents did not assent; of the 201 adolescents whose parents provided assent, 161 participated in the survey. We present data on these 161 adolescents. The age and gender of these adolescents were comparable to those of New York City in the 2000 Census (data not shown).
The Institutional Review Board of the New York Academy of Medicine reviewed and approved this study.

\section{Surveu Instrument}

Interviews were conducted using a structured questionnaire. First, we interviewed parents about their disaster experiences and mental health. Parents were considered to be directly affected by the attacks if they were inside the World Trade Center complex during the attacks, if they were injured during the attacks, if a friend or relative was killed during the attacks, if they lost possessions or property as a result of the attacks, if they lost a job due to the attacks, or if they were involved in the rescue efforts.

Probable PTSD in parents was assessed using a modified version of the National Women's Study (NWS) PTSD module (Resnick, Kilpatrick, Dansky, Saunders, \& Best, 1993). The NWS PTSD module assesses the presence of PTSD criteria B, C, and D symptoms and determines content for content-specific PTSD symptoms (e.g., content of dreams or nightmares). To measure probable PTSD related to the September 11 attacks, all reexperiencing symptoms (criterion B) and all content-specific avoidance symptoms (criterion C) were required to be related to the attacks.

Since its implementation, the NWS PTSD scale has been used in a number of large-scale mental health surveys involving over 16,000 completed telephone interviews (Galea et al., 2003; Hanson, Kilpatrick, Freedy, \& Saunders, 1995; Kilpatrick, Acierno, Resnick, Saunders, \& Best, 1997; Resnick, Kilpatrick, Dansky, Saunders, \& Best, 1993). The NWS PTSD module was externally validated in a field trial against the PTSD module of the Structured Clinical Interview for DSM-III-R (SCID) administered by mental health professionals (Kilpatrick et al., 1998; Spitzer, Williams, \& Gibbon, 1987). In the field trial, instrument sensitivity was $99 \%$ and specificity was $79 \%$ when compared to SCID diagnosis (Kilpatrick et al., 1998). In addition, this PTSD scale had a reported Kappa coefficient with the SCID of .71 for current PTSD (Kilpatrick et al., 1998; Spitzer, Williams, Gibbon, \& First, 1990). In studies conducted after the September 11 attacks, alpha coefficient for the NWS PTSD module 
ranged from .86 to .90 . The NWS PTSD module has also been compared to the PTSD checklist (PCL) (Blanchard, Jones-Alexander, Buckley, \& Forneris, 1996) in a random subsample of 229 participants in one of our surveys. The results of the comparison showed that the PCL had 75\% sensitivity and 95\% specificity in detecting PTSD cases as classified by our PTSD instrument (Boscarino et al., 2004). In a receiver operating characteristic analysis, against a PCL cutoff score of $>50$ (the optimal recommended cutoff), the PCL also optimally predicted PTSD using our instrument (area under the curve $=$.97) (Blanchard et al., 1996). Further details about the NWS PTSD module are available in a prior publication (Boscarino et al., 2004).

To assess probable parental depression since the attacks, we used a symptom scale consistent with DSM-IV (American Psychiatric Association, 1994) guidelines with symptoms from the SCID's major depressive episode interview from the non-patients interview schedule version (Spitzer et al., 1990), which has been used in other studies (Kilpatrick et al., 2003). Following DSM-IV guidelines, respondents met criteria for depression if they reported five or more symptoms for at least 2 weeks. For further details regarding the measures used in this study, we refer the reader to previous publications (Galea et al., 2002; Galea et al., 2003).

Demographic information was obtained from the parent including the race (White, Black, Hispanic, Other) and gender of the adolescent, the annual household income before taxes $(<\$ 30,000 ; \$ 30,000-75,000 ;>\$ 75,000)$, whether the adolescent had siblings, and whether it was a single-parent household. We also asked parents about previous stressful life events in the adolescent's life, such as a natural disaster or an accident by modifying an existing measure that asks parents if the child had ever experienced any of eight possible stressful life events (Stamm, 1996). The number of previous stressful life events experienced by the adolescent was summed to create a stressful life events scale with scores ranging from 0 to 8 . Because of the relatively small number of adolescents experiencing more than three stressful prior life events, this variable was collapsed into two categories with fewer than three stressful life events coded as 1 and three or more life events coded as 2 .
We also interviewed adolescents directly. Social support available to the adolescents including emotional and instrumental support was assessed with two questions: "In the six months before the World Trade Center disaster, when you needed someone to talk to about a personal problem, how often were each of the following people available?" and "In the six months before the World Trade Center disaster, when you needed money and other things, how often were each of the following people available?" We assessed levels of emotional and instrumental support provided by close friends, fathers, mothers, other adults close to the adolescent, siblings, and teachers. Two variables, one for emotional and one for instrumental support, were created by summing the level of support available from each person; each ranged from 0 to 24 . These scales have been previously validated in a sample of adolescents (Seidman et al., 1995). Based on this validation study, a score of 8 or less was coded as low support, a score of 9 to 16 was coded as medium support, and a score of 17 or greater was coded as high support.

Adolescents were queried about their disaster experiences. They were asked if they saw the attacks in person, if they had to leave school early on September 11, if they were concerned about their own safety during the attacks, and if they had seen a parent or another close adult cry about the events. The approximate number of hours per day the adolescent watched television in the week after the attacks was also ascertained and coded as $<2$ hours or $>2$ hours.

We asked adolescents about panic symptoms they experienced using a modified version of the Diagnostic Interview Schedule (DIS) for panic attacks (Centers for Disease Control, 1989) phrased to assess peri-event symptoms that occurred in the first few hours after the events of September 11. The presence of at least four symptoms established a probable diagnosis of a peri-event panic attack (American Psychiatric Association, 1994).

We assessed posttraumatic stress reactions in adolescents using the UCLA PTSD Index for DSM-IV (Adolescent Version), which was designed to quickly and efficiently screen adolescents who have experienced a traumatic event. This instrument is not intended to replace a structured clinical interview needed to make a definitive PTSD 
diagnosis. The 22 items measured current PTSD symptoms on a 4-point scale ranging from none of the time to most of the time. The index clusters on three subscales: criterion B (reexperiencing), C (avoidance/numbing), and D (hyperarousal). Internal consistency for the three subscales ranges from .67 to .82 and agreement with structured clinician interviews has been demonstrated $66 \%$ to $83 \%$ of the time (Rodriguez, Steinberg, \& Pynoos, 2002). Adolescent respondents who reported one of five possible criterion $\mathrm{B}$ symptoms, three of nine possible criterion $\mathrm{C}$ symptoms, and two of six possible criterion $\mathrm{D}$ symptoms qualified for a probable diagnosis of PTSD. They were considered positive for probable subthreshold PTSD if they met criteria $\mathrm{B}$ and $\mathrm{C}$, criteria $\mathrm{B}$ and $\mathrm{D}$, or criteria $\mathrm{C}$ and $\mathrm{D}$ (Pynoos, Rodriguez, Steinberg, Stuber, \& Frederick, 1998). Fear that the traumatic event would reoccur was also assessed.

\section{Statistical Analusis}

We describe the prevalence of adolescent disaster experiences, reactions to the attacks, and social support as reported by adolescents. We also describe the prevalence of the adolescent's prior stressful life events and demographic characteristics, parental mental health problems (including probable PTSD and probable depression), and if the parent was directly affected by the attacks as reported by parents. We show bivariate relationships between the covariates and probable PTSD and probable subthreshold PTSD among adolescents. Sampling weights were developed and applied to the data to adjust for sampling fraction and to correct potential selection bias related to the number of telephones and children in the household. Standard errors were estimated using SUDAAN software to account for the complex sampling design (Shah, Barnewell, \& Bieler, 1997).

\section{RESULTS}

Table 1 provides a description of the adolescents who participated in the study. The sample included approximately equal numbers of boys and girls and the majority was White.
Most adolescents (89.0\%) experienced fewer than three stressful past life events. Adolescents reported receiving higher levels of emotional as compared to instrumental support with only $20.6 \%$ reporting low levels of emotional support and $34.1 \%$ reporting low levels of instrumental support in the 6 months before the attacks.

Over one half $(56.7 \%)$ of the adolescents left school early on September 11;57.7\% were concerned about their safety on the day of the attacks; and $50.9 \%$ saw a parent or another adult close to them cry about the attacks. Approximately one third $(34.4 \%)$ of the adolescents reported watching on average 2 or more hours of television per day in the week after the attacks.

In reaction to the events, $16.4 \%$ of adolescents met criteria for a probable peri-event panic attack, $12.6 \%$ met criteria for probable PTSD, and $26.2 \%$ met criteria for probable subthreshold PTSD. Only 5.2\% of parents met criteria for probable PTSD.

Table 2 displays factors associated with probable PTSD and probable subthreshold PTSD in bivariate analyses using a significance level of $p<.05$. Living in a single-parent household and having experienced a probable peri-event panic attack on September 11 were associated with probable PTSD. Having had a peri-event panic attack was associated with probable subthreshold PTSD.

\section{DISCUSSION}

Of adolescents surveyed, $12.6 \%$ reported symptoms consistent with probable PTSD 6 to 9 months after the September 11 terrorist attacks, with an additional 26.2\% meeting study criteria for probable subthreshold PTSD. Subthreshold PTSD appears to be common in adults and to carry with it potential for clinically significant impairment (Marshall et al., 2001; Moreau \& Zisook, 2003) with a risk of disability approaching, if not matching, that of PTSD (Stein, Walker, Hazen, \& Forde, 1997). Exposure to trauma, even when it does not result in PTSD, is also associated with emotional and behavioral problems, poor academic performance, and impact on health in adolescents (Giaconia et al., 1995). Thus, our results suggest the potential for emotional and behavioral problems 
Table 1. Description of Study Population $(N=161)$

\begin{tabular}{|c|c|c|}
\hline Variable and value & $n$ & Weighted \% \\
\hline \multicolumn{3}{|l|}{ Demographic characteristics of adolescent ${ }^{\mathrm{a}}$} \\
\hline \multicolumn{3}{|l|}{ Gender } \\
\hline Male & 84 & 52.8 \\
\hline Female & 75 & 47.2 \\
\hline \multicolumn{3}{|l|}{ Race } \\
\hline White & 80 & 54.0 \\
\hline Non-White & 79 & 46.0 \\
\hline \multicolumn{3}{|l|}{ Annual income before taxes } \\
\hline Under $\$ 30,000$ & 49 & 38.7 \\
\hline$\$ 30,000-\$ 75,000$ & 49 & 33.5 \\
\hline$\$ 75,000+$ & 47 & 29.8 \\
\hline Single-parent household & 61 & 39.2 \\
\hline Adolescent has siblings & 90 & 75.1 \\
\hline \multicolumn{3}{|l|}{ Adolescent's exposure to the September 11 attacks ${ }^{\mathrm{b}}$} \\
\hline Left school early & 89 & 56.7 \\
\hline Saw the attacks in person & 22 & 10.2 \\
\hline \multicolumn{3}{|c|}{ Average television viewing per day the week after the attacks } \\
\hline Less than 2 hours & 73 & 65.6 \\
\hline 2 or more hours & 41 & 34.4 \\
\hline Was concerned about his/her safety & 90 & 57.7 \\
\hline Saw a parent or another close adult cry & 79 & 50.9 \\
\hline \multicolumn{3}{|l|}{ Adolescent's reaction to the event ${ }^{b}$} \\
\hline Had a panic attack on September 11 & 30 & 16.4 \\
\hline Subthreshold posttraumatic stress disorder (PTSD) & 36 & 26.2 \\
\hline Probable PTSD & 12 & 12.6 \\
\hline \multicolumn{3}{|l|}{ Adolescent's social support ${ }^{b}$} \\
\hline \multicolumn{3}{|l|}{ Emotional support } \\
\hline High & 61 & 36.3 \\
\hline Medium & 66 & 43.2 \\
\hline Low & 31 & 20.6 \\
\hline \multicolumn{3}{|l|}{ Monetary and other support } \\
\hline High & 25 & 22.8 \\
\hline Medium & 62 & 43.2 \\
\hline Low & 48 & 34.1 \\
\hline Adolescent experienced 3 or more stressful life events ${ }^{b}$ & 15 & 11.0 \\
\hline \multicolumn{3}{|l|}{ Parental exposure and reaction to the attacks ${ }^{\mathrm{a}}$} \\
\hline Directly affected by the attacks & 42 & 22.9 \\
\hline Probable PTSD & 16 & 5.2 \\
\hline Probable depression & 20 & 8.7 \\
\hline Probable PTSD or depression & 25 & 10.1 \\
\hline
\end{tabular}

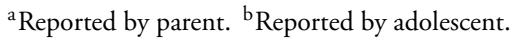

and impairment in many adolescents following the World Trade Center attacks.

A probable peri-event panic attack was reported by $16.4 \%$ of the adolescents in this sample. The strength of the association of a probable peri-event panic attack with probable PTSD and probable subthreshold PTSD in this study adds to the developing literature on panic and PTSD (Falsetti et al., 1995; Leskin \& Sheikh, 2002). This 
Table 2. Bivariate Relationships Between Predictor Variables and Subthreshold Posttraumatic Stress Disorder (PTSD) $(n=36)$ or Probable PTSD $(n=12)$

\begin{tabular}{|c|c|c|c|c|}
\hline & \multicolumn{2}{|c|}{ Subthreshold PTSD ${ }^{c}$} & \multicolumn{2}{|c|}{$\mathrm{PTSD}^{\mathrm{c}}$} \\
\hline & OR & CI & OR & CI \\
\hline \multicolumn{5}{|c|}{ Demographic characteristics of adolescent ${ }^{\mathrm{a}}$} \\
\hline \multicolumn{5}{|c|}{ Gender } \\
\hline Male & - & - & & \\
\hline Female & 1.30 & $(0.50,3.35)$ & 1.24 & $(0.31,4.94)$ \\
\hline \multicolumn{5}{|l|}{ Race } \\
\hline White & - & - & & \\
\hline Non-White & 1.37 & $(0.52,3.58)$ & 1.99 & $(0.52,7.64)$ \\
\hline \multicolumn{5}{|c|}{ Annual income before taxes } \\
\hline Under $\$ 30,000$ & 1.79 & $(0.55,5.80)$ & 4.19 & $(0.86,20.46)$ \\
\hline$\$ 30,000-\$ 75,000$ & - & - & - & - \\
\hline$\$ 75,000+$ & 1.73 & $(0.58,5.18)$ & 0.95 & $(0.14,6.70)$ \\
\hline \multicolumn{5}{|c|}{ Single-parent household } \\
\hline No & - & - & - & - \\
\hline Yes & 1.94 & $(0.75,5.01)$ & $4.77^{*}$ & $(1.24,18.33)$ \\
\hline \multicolumn{5}{|l|}{ Adolescent has siblings } \\
\hline No & - & - & - & - \\
\hline Yes & 0.66 & $(0.27,1.61)$ & 1.10 & $(0.29,4.21)$ \\
\hline \multicolumn{5}{|c|}{ Adolescent's exposure to the September 11 attacks ${ }^{b}$} \\
\hline \multicolumn{5}{|c|}{ Left school early } \\
\hline No & - & - & - & - \\
\hline Yes & 0.81 & $(0.31,2.13)$ & 1.32 & $(0.33,5.29)$ \\
\hline \multicolumn{5}{|c|}{ Saw the attacks in-person } \\
\hline No & - & - & - & - \\
\hline Yes & 1.31 & $(0.32,5.37)$ & 0.40 & $(0.05,3.51)$ \\
\hline \multicolumn{5}{|c|}{ Average television viewing per day the week after the attacks } \\
\hline Less than 2 hours & - & - & - & - \\
\hline 2 or more hours & 2.44 & $(0.78,7.64)$ & 3.16 & $(0.63,15.95)$ \\
\hline \multicolumn{5}{|c|}{ Was concerned about his/her safety } \\
\hline No & - & - & - & - \\
\hline Yes & 2.29 & $(0.88,6.00)$ & 2.78 & $(0.68,11.27)$ \\
\hline \multicolumn{5}{|c|}{ Saw a parent or another close adult cry } \\
\hline No & - & - & - & - \\
\hline Yes & 0.40 & $(0.14,1.13)$ & 0.54 & $(0.12,2.50)$ \\
\hline \multicolumn{5}{|c|}{ Adolescent's reaction to the event, social support, and life events ${ }^{b}$} \\
\hline \multicolumn{5}{|c|}{ Had a panic attack on September 11} \\
\hline No & - & - & - & - \\
\hline Yes & $9.57^{*}$ & $(3.16,29.0)$ & $10.54^{*}$ & $(2.57,43.15)$ \\
\hline \multicolumn{5}{|l|}{ Emotional support } \\
\hline High & - & - & - & - \\
\hline Medium & 3.45 & $(1.15,10.36)$ & 5.13 & $(0.95,27.74)$ \\
\hline Low & 2.80 & $(0.76,10.33)$ & 1.20 & $(0.15,9.49)$ \\
\hline
\end{tabular}


Table 2. (Continued)

\begin{tabular}{|c|c|c|c|c|}
\hline & \multicolumn{2}{|c|}{ Subthreshold PTSD ${ }^{c}$} & \multicolumn{2}{|c|}{$\mathrm{PTSD}^{\mathrm{c}}$} \\
\hline & OR & $\mathrm{CI}$ & OR & $\mathrm{CI}$ \\
\hline \multicolumn{5}{|c|}{ Monetary and other support } \\
\hline High & - & - & - & - \\
\hline Medium & 2.03 & $(0.52,7.91)$ & 2.10 & $(0.36,12.12)$ \\
\hline Low & 0.98 & $(0.23,4.21)$ & 0.14 & $(0.01,1.74)$ \\
\hline \multicolumn{5}{|c|}{ Adolescent has experienced 3 or more stressful life events ${ }^{b}$} \\
\hline No & - & - & - & - \\
\hline Yes & 2.44 & $(0.55,10.78)$ & 0.70 & $(0.08,6.47)$ \\
\hline \multicolumn{5}{|c|}{ Parental exposure and reaction to the attacks ${ }^{a}$} \\
\hline \multicolumn{5}{|c|}{ Directly affected by the attacks } \\
\hline No & - & - & - & - \\
\hline Yes & 1.09 & $(0.39,3.06)$ & 0.32 & $(0.04,2.76)$ \\
\hline \multicolumn{5}{|c|}{ Probable PTSD } \\
\hline No & - & - & - & - \\
\hline Yes & 1.52 & $(0.33,7.02)$ & 3.15 & $(0.55,18.18)$ \\
\hline \multicolumn{5}{|c|}{ Probable depression } \\
\hline No & - & - & - & - \\
\hline Yes & 2.33 & $(0.60,9.02)$ & 2.90 & $(0.49,17.27)$ \\
\hline \multicolumn{5}{|c|}{ Probable PTSD or depression } \\
\hline No & - & - & - & - \\
\hline Yes & 2.95 & $(0.88,9.88)$ & 3.90 & $(0.82,18.59)$ \\
\hline
\end{tabular}

Note. Subthreshold PTSD cases were compared to adolescents below the criteria for subthreshold PTSD. PTSD cases were compared to adolescents below the criteria for PTSD.

${ }^{a}$ Reported by parent. ${ }^{b}$ Reported by adolescent. ${ }^{\mathrm{c}}$ There were 36 cases of subthreshold PTSD and 12 cases of PTSD.

$* p<.001$ (2-tailed $\chi^{2}$ test).

relationship may represent the interplay of a predisposition to anxiety with the severity of trauma exposure or with the cognitive appraisal of threat (Asarnow et al., 1999; Lonigan et al., 1994), and it may have implications for the recovery and future mental health of these adolescents. Although the reactions we describe as probable peri-event panic attacks may represent a normal or extreme fear reaction or the subjective reaction required by the stressor criterion for a diagnosis of PTSD, the presence of these reactions are potentially important markers to help identify those with vulnerability to adverse outcomes to trauma exposure. The presence of these attacks may have implications for longer-term prognosis of either PTSD or panic and they also provide an opportunity for early intervention.

Our results raise issues about psychiatric nosology that have recently been the subject of some discussion in the posttraumatic stress literature (North \& Pfefferbaum,
2002). Specifically, our results leave unanswered the extent to which the designation of probable peri-event panic attack in this study represented evidence of a panic attack rather than normal fear responses or symptoms of acute stress disorder. Diagnosable disorders and diagnostic criteria within psychiatry have not been subjected to extensive validation. Rounsaville and colleagues (2002) have addressed the uncertainties of validating psychiatric diagnoses, which assumes that "psychiatric disorders are discrete biomedical entities with clear phenotypic boundaries" (p. 8). Acknowledging this concern, the DSM-IV-TR (American Psychiatric Association, 2000, p. xxxii) states that diagnostic criteria are "meant to serve as guidelines to be informed by clinical judgment." Thus, it is reasonable to expect and allow some imprecision in the application of diagnostic criteria in general as well as in our study. 
Specific issues related to both panic attacks and PTSD reinforce the difficulties in describing and studying these conditions. The DSM-IV-TR (2000) notes that a panic attack is not itself a "codable disorder" (p. 432) and that it may occur in the context of any anxiety disorder as well as other mental or general medical conditions. In addition, psychiatric nomenclature allows overlap in symptoms among various disorders perhaps most prominently among the anxiety and affective conditions. Furthermore, diagnostic criteria for PTSD are relatively new in the history of psychiatric classification with flux in the criteria over successive iterations of the diagnostic manual. The stressor criterion has been modified to address the qualifying trauma itself and the subjective initial reaction of "intense fear, helplessness, or horror" (American Psychiatric Association, 2000, p. 428), which was absent from manuals prior to the fourth edition.

Finally, prior studies have also evidenced the congruence between the required subjective element of the PTSD stressor criterion and panic. In a field trial evaluation of the PTSD construct, Kilpatrick and colleagues (1998) found that a "panic-physiological arousal" factor, which included nine panic attack symptoms accounted for almost 40\% of the variance in the subjective distress component of the stressor criterion. The second largest factor included additional symptoms of a panic attack and helplessness, confusion, surprise, and fear. Thus, future studies that use comprehensive assessment tools and longitudinal design will be needed to further consider the nature of peri-event reactions, to clarify the association between these reactions and PTSD, and to examine the course and pathogenic pathways for the development of panic, PTSD, and other anxiety disorders.

Our results also raise methodological issues. We were unable in this lay-administered questionnaire-based interview to query comprehensively aspects of these adolescents' peri-event reactions that might have distinguished panic from normal fear. Questionnaire design may increase falsepositive responses (Hayward et al., 1997). In addition, Hayward and colleagues (1997) found low test-retest reliability in their measure of panic in a young adolescent sample studied at one-year follow-up. Our sample included some older adolescents in whom reliability may be greater, but this issue deserves attention in future studies. We also did not assess prior or subsequent anxiety sensitivity, panic attacks, or other anxiety conditions, which might have provided important information on the clinical implications of our findings.

Because of the time and space constraints in the survey, we were unable to measure many child, family, or social variables that would have enhanced the study. Future studies should assess functioning alongside PTSD symptoms to determine the clinical significance of PTSD symptomatology in adolescents. It is unclear whether we would have obtained a better assessment of the stressful life events of adolescents had we asked them directly instead of asking their parents. Due to power limitations, we were unable to explore other factors that may be related to probable PTSD and probable subthreshold PTSD including factors that may mediate the relation of probable peri-event panic with probable PTSD and probable subthreshold PTSD.

A final concern is the cooperation rate for the adolescent survey that was based on the adult cooperation rate for the main survey (56\%), the proportion of adults who gave permission for adolescents to be contacted (85\%), and the cooperation rate for the adolescents themselves (75\%). Attrition occurred at each stage resulting in a lower overall cooperation rate for the final adolescent survey (35.7\%) than the adult survey, thus raising questions about the generalizability of the findings. This staged procedure is standard methodology for telephone survey research with adolescents, and cooperation rates for each successive portion of the survey were acceptable and consistent with other telephone surveys of adolescents (Hemenway \& Miller, 2004).

\section{CONCLUSIONS}

The strong relationship between a probable peri-event panic attack and later probable PTSD and probable subthreshold PTSD underscores the need to assess adolescents early in the aftermath of major traumatic events. Although we cannot be certain that the probable peri-event panic reactions experienced by some of the participants in this study were not merely extreme peri-event fear reactions, 
our results, in the context of current literature describing the frequency of panic attacks in this age group and their association with distress and morbidity (Black \& Robbins, 1990; Ollendick, 1998; Ollendick et al., 1994), suggest the importance of attention to this issue in future work. Findings related to nosology may appear unimportant on first examination but they have the potential to shape our understanding of illness and its prognosis and treatment. Future studies should use longitudinal design and more comprehensive assessment measures to examine the course of, and relationships among, anxiety disorders in adolescents exposed to traumatic events.

\section{REFERENCES}

American Association for Public Opinion Research. (2000). Standard definitions: Final dispositions of case codes and outcome rates for surveys. Ann Arbor, MI: AAPOR. Retrieved May 11, 2004 from http://www.aapor.org/default.asp?page=survey methods/standards_and_best_practices/standard_definitions ${ }^{\text {c }}$

American Psychiatric Association. (1994). Diagnostic and statistical manual of mental disorders (4th ed.). Washington, DC: Author.

American Psychiatric Association. (2000). Diagnostic and statistical manual of mental disorders (4th TR ed.). Washington, DC: Author.

Asarnow, J., Glynn, S., Pynoos, R. S., Nahum, J., Guthrie, D., Cantwell, D. P., et al. (1999). When the Earth stops shaking: Earthquake sequelae among children diagnosed for pre-earthquake psychopathology. Journal of the American Academy of Child and Adolescent Psychiatry, 38, 10161023.

Barlow, D. H. (2002). Anxiety and its disorders: The nature and treatment of anxiety and panic (2nd ed.) (pp. 105-138). New York: The Guilford Press.

Black, B., \& Robbins, D. R. (1990). Panic disorder in children and adolescents. Journal of the American Academy of Child and Adolescent Psychiatry, 29, 36-44.

Blanchard, E. B., Jones-Alexander, J., Buckley, T. C., \& Forneris, C. A. (1996). Psychometric properties of the PTSD checklist. Behavior Research and Therapy, 34, 669-673.

Boscarino, J. A., Galea, S., Adams, R. E., Ahern, J., Resnick, H., $\&$ Vlahov, D. (2004). Mental health service and medication use in New York City after the September 11, 2001, terrorist attack. Psychiatric Services, 55(3), 274-283.
Breton, J., Valla, J., \& Lambert, J. (1993). Industrial disaster and mental health of children and their parents. Journal of the American Academy of Child and Adolescent Psychiatry, 32, 438-445.

Centers for Disease Control and Prevention. (1989). Diagnostic Interview Schedule (DIS). In health status of Vietnam veterans. Supplement C: Medical and psychological procedure manuals and forms (pp. 405-499). Atlanta, GA: Author.

Earls, F., Smith, E., Reich, W., \& Jung, K. G. (1988). Investigating psychopathological consequences of a disaster in children: A pilot study incorporating a structured diagnostic interview. Journal of the American Academy of Child and Adolescent Psychiatry, 27, 90-95.

Fairbrother, G., Stuber, J., Galea, S., Fleischman, A. R., \& Pfefferbaum, B. (2003). Posttraumatic stress reactions in New York City children after the September 11, 2001, terrorist attacks. Ambulatory Pediatrics, 3(6), 304-311.

Falsetti, S. A., Resnick, H. S., Dansky, B. S., Lydiard, R. B., \& Kilpatrick, D. G. (1995). The relationship of stress to panic disorder: Cause or effect? In C. M. Mazure (Ed.), Does stress cause psychiatric illness? (pp. 111-147). Washington, DC: American Psychiatric Press, Inc.

Galea, S., Ahern, J., Resnick, H., Kilpatrick, D., Bucuvalas, M., Gold, J., et al. (2002). Psychological sequelae of the September 11 terrorist attacks in New York City. New England Journal of Medicine, 346, 982-987.

Galea, S., Vlahov, D., Resnick, H., Ahern, J., Susser, E., Gold, J., et al. (2003). Trends of probable post-traumatic stress disorder in New York City after the September 11 terrorist attacks. American Journal of Epidemiology, 158, $514-524$.

Giaconia, R. M., Reinherz, H. Z., Silverman, A. B., Pakiz, B., Frost, A. K., \& Cohen, E. (1995). Traumas and posttraumatic stress disorder in a community population of older adolescents. Journal of the American Academy of Child and Adolescent Psychiatry, 34, 1369-1380.

Green, B. L., Korol, M., Grace, M. C., Vary, M. G., Leonard, A. C., Gleser, G. C., et al. (1991). Children and disaster: Age, gender, and parental effects on PTSD symptoms. Journal of the American Academy of Child and Adolescent Psychiatry, 30, 945-951.

Hanson, R. F., Kilpatrick, D. G., Freedy, J. R., \& Saunders, B. E. (1995). Los Angeles County after the 1992 civil disturbances: Degree of exposure and impact on mental health. Journal of Consulting and Clinical Psychology, 63, 987-996.

Hayward, C., Killen, J. D., Kraemer, H. C., Blair-Greiner, A., Strachowski, D., Cunning, D., et al. (1997). Assessment and phenomenology of nonclinical panic attacks in adolescent girls. Journal of Anxiety Disorders, 11, 17-32. 
Hayward, C., Killen, J. D., Kraemer, H. C., \& Taylor, C. B. (2000). Predictors of panic attacks in adolescents. Journal of the American Academy of Child and Adolescent Psychiatry, 39, 207-214.

Hemenway, D., \& Miller, M. (2004). Gun threats against and selfdefense gun use by California adolescents. Archives of Pediatrics \& Adolescent Medicine, 158, 395-400.

Jones, J. C., \& Barlow, D. H. (1990). The etiology of posttraumatic stress disorder. Clinical Psychology Review, 10, 299-328.

Kilpatrick, D. G., Acierno, R., Resnick, H. S., Saunders, B. E., \& Best, C. L. (1997). A 2-year longitudinal analysis of the relationships between violent assault and substance use in women. Journal of Consulting and Clinical Psychology, 65(5), 834-847.

Kilpatrick, D. G., Resnick, H. S., Freedy, J. R., Pelcovitz, D., Resick, P., Roth, S., et al. (1998). Posttraumatic stress disorder field trial: Evaluation of the PTSD construct-Criteria A through E. In T. A. Widiger, A. J. Frances, H. A. Pincus, R. Ross, \& M. B. First (Eds.), DSM-IV Sourcebook (Vol. 4) (pp. 803-838). Washington, DC: American Psychiatric Association.

Kilpatrick, D. G., Ruggiero, K. J., Acierno, R., Saunders, B. E., Resnick, H. S., \& Best, C. L. (2003). Violence and risk of PTSD, major depression, substance abuse/dependence, and comorbidity: Results from the National Survey of Adolescents. Journal of Consulting and Clinical Psychology, 71, 692-700.

La Greca, A. M., Silverman, W. K., Vernberg, E. M., \& Prinstein, M. J. (1996). Symptoms of posttraumatic stress in children after Hurricane Andrew: A prospective study. Journal of Consulting and Clinical Psychology, 64, 712-723.

Leskin, G. A., \& Sheikh, J. I. (2002). Lifetime trauma history and panic disorder: Findings from the National Comorbidity Survey. Anxiety Disorders, 16, 599-603.

Lonigan, C. J., Shannon, M. P., Taylor, C. M., Finch, A. J., Jr., \& Sallee, F. R. (1994). Children exposed to disaster: II. Risk factors for the development of post-traumatic symptomatology. Journal of the American Academy of Child and Adolescent Psychiatry, 33, 94-105.

Marshall, R. D., Olfson, M., Hellman, F., Blanco, C., Guardino, M., \& Struening, E. L. (2001). Comorbidity, impairment, and suicidality in subthreshold PTSD. American Journal of Psychiatry, 158, 1467-1473.

McFarlane, A. C. (1987). Posttraumatic phenomena in a longitudinal study of children following a natural disaster. Journal of the American Academy of Child and Adolescent Psychiatry, 26, 764-769.

Moreau, C., \& Zisook, S. (2003). Rationale for a posttraumatic stress spectrum disorder. FOCUS, 1, 265-272.
North, C. S., \& Pfefferbaum, B. (2002). Research on the mental health effects of terrorism. Journal of the American Medical Association, 288, 633-636.

Ollendick, T. H. (1998). Panic disorder in children and adolescents: New developments, new directions. Journal of Clinical Child Psychiatry, 27, 234-245.

Ollendick, T. H., Mattis, S. G., \& King, N. J. (1994). Panic in children and adolescents: A review. Journal of Child Psychology and Psychiatry, 35, 113-134.

Pfefferbaum, B., Doughty, D. E., Reddy, C., Patel, N., Gurwitch, R. H., Nixon, S. J., et al. (2002). Exposure and peritraumatic response as predictors of posttraumatic stress in children following the 1995 Oklahoma City bombing. Journal of Urban Health, 79, 354-363.

Pfefferbaum, B., Nixon, S. J., Krug, R. S., Tivis, R. D., Moore, V. L., Brown, J. M., et al. (1999). Clinical needs assessment of middle and high school students following the 1995 Oklahoma City bombing. American Journal of Psychiatry, 156, 1069-1074.

Pitman, R. K. (1993). Biological findings in posttraumatic stress disorder: Implications for DSM-IV classification. In J. R. T. Davidson \& E. B. Foa (Eds.), Posttraumatic stress disorder: DSM-IV and beyond (pp. 173-189). Washington, DC: American Psychiatric Press, Inc.

Pynoos, R., Rodriguez, N., Steinberg, A., Stuber, M., \& Frederick, C. (1998). UCLA Adolescent PTSD Index 1. Los Angeles, CA: UCLA Trauma Psychiatry Service.

Resnick, H. S., Kilpatrick, D. G., Dansky, B. S., Saunders, B. E., \& Best, C. L. (1993). Prevalence of civilian trauma and posttraumatic stress disorder in a representative national survey of women. Journal of Consulting and Clinical Psychology, 61, 984-991.

Rodriguez, N., Steinberg, A., \& Pynoos, R. S. (2002, November). Preliminary psychometric analysis of the child and parent report versions. Paper presented at the International Society for Traumatic Stress Studies, New Orleans, LA.

Rounsaville, B. J., Alarcón, R. D., Andrews, G., Jackson, J. S., Kendell, R. E., \& Kendler, K. (2002). Basic nomenclature issues for DSM-V. In D. J. Kupfer, M. B. First, \& D. A. Regier (Eds.), A research agenda for DSM-V (pp. 1-29). Washington, DC: American Psychiatric Association.

Schuster, M. A., Stein, B. D., Jaycox, L. H., Collins, R. L., Marshall, G. N., Elliott, M. N., et al. (2001). A national survey of stress reactions after the September 11, 2001, terrorist attacks. New England Journal of Medicine, 345, 1507-1512.

Schwarz, E. D., \& Kowalski, J. M. (1991). Malignant memories: PTSD in children and adults after a school shooting. Journal of the American Academy of Child and Adolescent Psychiatry, 30, 936-944. 
Seidman, E., Allen, L., Aber, J. L., Mitchell, C., Feinman, J., Yoshikawa, H., et al. (1995). Development and validation of adolescent-perceived microsystem scales: Social support, daily hassles, and involvement. American Journal of Community Psychology, 23, 355-388.

Shah, B., Barnewell, B., \& Bieler, G. (1997). SUDAAN users manual, Release 7.5. Research Triangle Park, NC: Research Triangle Institute.

Spitzer, R. L., Williams, J. B., Gibbon, M., \& First, M. B. (1990). Structured Clinical Interview for DSM-III-R. Washington, DC: American Psychiatric Press.
Stamm, B. H. (1996). Contextualizing death and trauma: A preliminary endeavor. In C. R. Figley (Ed.), Death and trauma (pp. 3-21). New York: Brunner/Mazel.

Stein, M. B., Walker, J. R., Hazen, A. L., \& Forde, D. R. (1997). Full and partial posttraumatic stress disorder: Findings from a community survey. American Journal of Psychiatry, 154, 11141119 .

Udwin, O., Boyle, S., Yule, W., Bolton, D., \& O'Ryan, D. (2000). Risk factors for long-term psychological effects of a disaster experienced in adolescence: Predictors of post traumatic stress disorder. Journal of Child Psychology and Psychiatry, 41, 969-979. 